\title{
Medium range forecasts using cut-cells: a sensitivity study
}

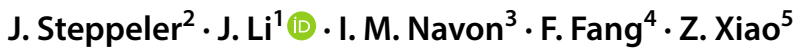

Received: 20 January 2019 / Accepted: 21 June 2019 / Published online: 3 July 2019

(c) The Author(s) 2019

\begin{abstract}
In this study, 10-day forecasts of Atlantic lows are investigated by comparing the forecasts of the standard Climate version of the Lokal Modell using terrain-following coordinates to that of the cut-cell Lokal Modell with $z$ coordinates (LMZ). As indicated by idealized tests of the atmosphere at rest and mountain wave tests, LMZ exhibits a much better approximation of the lower boundary condition due to the use of the cut-cell approximation. An important difference to the terrain-following coordinate is that with cut-cells, a single steep mountain as defined different from 0 at only one point, has a reasonable up-downwind scheme and does not become unstable. We are interested in the large-scale structure of Atlantic lows, which are under the influence of the high orography of Greenland. The steep orography of Greenland has a considerable impact on these lows, even though they are positioned to a large part over water. It is found that at Day 6, both models yield similar forecasts, which is consistent with the fact that Day 6 is in the useful forecast range of both models. At Day 10, the forecasts are quite different, indicating a sensitivity of the 10-day forecast to the discretization in question.
\end{abstract}

\section{Introduction}

Numerical weather forecasts are mostly based on models using terrain-following coordinates. Problems of this approximation are pointed out by Sundquist (1967). One error encountered is that the atmosphere at rest for a horizontally stratified atmosphere is not represented well (Sundquist 1967; Steppeler et al. 2006). In meso-scale models with steep orography, this error is more relevant. Although modifications of the terrain-following atmosphere have reduced this error, this error is still very severe (Good et al. 2014).

Responsible Editor: C. Simmer.

J. Li

ljx2311@mail.iap.ac.cn

1 International Center for Climate and Environment Sciences, Institute of Atmospheric Physics, Chinese Academy of Sciences, Beijing 100029, China

2 Climate Service Center Germany (GERICS), Fischertwiete 1, 20095 Hamburg, Germany

3 Department of Scientific Computing, Florida State University, Tallahassee, FL 32306-4120, USA

4 Applied Modelling and Computation Group, Department of Earth Science and Engineering, Imperial College London, Prince Consort Road, London SW7 2AZ, UK

5 Guangxi Meteorological Observatory, Nanning 530022, Guangxi, China
New tests for the numerical errors associated with the representation of the lower boundary have been investigated (Good et al. 2014; Shaw and Weller 2016; Steppeler and Klemp 2017; Steppeler et al. 2019). It is also known that there is a large sensitivity of forecasts in the range up to 10 days on the choice of the orography (Wallace et al. 1983). For a discussion of these developments see Steppeler et al. (2006) and Li et al. (2018). For improvements of the orographic representation with terrain-following models, see Schär et al. (2002), Leuenberger et al. (2010) and Klemp (2011).

The cut-cell method (Adcroft et al. 1997; Steppeler et al. 2002, 2008; Good et al. 2014; Shaw and Weller 2016), based on a height coordinate, is an approach for representing terrains by a bilinear spline. The computational cells are cut by the terrains. The errors of older cut-cell approaches demonstrated by Gallus and Klemp (2000) have concerned artificial flow separation and non-convergence. Gallus (2000) showed that the drawbacks shown in artificial tests had translated into deficiencies of local forecasts in the older version of the ETA model (Mesinger et al. 1988). However, cut-cell models are now able to avoid the errors of representing the resting atmosphere by a thin-wall approximation and a cellmerging technique (Steppeler et al. 2006; Yamazaki and Satomura 2008; Lock et al. 2012; Yamazaki et al. 2016). The Lokal Modell with $z$ coordinates (LMZ, Steppeler et al. 2006) could perform realistic forecasts using observed initial 
data. Steppeler et al. (2006) showed that the 24-h forecast of meso-scale features was considerably improved by cut-cells and the root mean square error of temperature after $24 \mathrm{~h}$ was reduced. An example of 5-day forecasts and the improvement of precipitation was given in Steppeler et al. (2013). Mesinger and Veljovic (2017) reported that the problems of artificial flow separation and non-convergence demonstrated by Gallus and Klemp (2000) have been reduced by the recent modification of the ETA model. These changes in ETA model were partially following Steppeler et al. (2002) (see details in Li et al. 2019). The modifications are described by Mesinger et al. (2012) who described the relation to Steppeler et al. (2002) as simplifications resulting into a "poor man's cut-cell scheme". The flow around the mountain tested by Gallus and Klemp (2000) has an analytic solution (Long's solution). For a shallow and smooth mountain, this is reproduced well by the terrain-following scheme of Gallus and Klemp (2000) and the cut-cell scheme of Steppeler et al. (2002). Gallus and Klemp (2000) gave a solution of the ETA cut-cells with a better representation of the vorticity at mountain edges which avoided some, but not all of the errors seen with the ETA system and this solution will be called Klemp's improved solution. It should be noted that Klemp's improved solution still shows considerable errors as compared to Long's solution. The maximum of the $u$ field near $1.5 \mathrm{~km}$ height is too weak and the flow at the surface is too weak. The improved ETA result, as presented by Mesinger and Veljovic (2017) showed further deficiencies. The maximum of $u$ is now on the surface, rather than $1.5 \mathrm{~km}$ above. And the artificial maximum on the surface has a strong amplitude. Above the surface the improved ETA solution looks like a smoothed version of Klemp's improved solution. It is unknown if the introduction of more diffusion plays a role in the improvements reported by Mesinger and Veljovic (2017). Both methods, Mesinger et al. (2012) and Steppeler et al. (2002), involved numerical diffusion. Currently it remains uncertain whether the remaining errors of the solutions of Mesinger and Veljovic (2017) are caused by a less smooth mountain representation with the "poor man's cut-cell" approach (Mesinger et al. 2012) and whether the errors mentioned have an impact on forecasts (Gallus 2000). For the revised ETA model (Mesinger and Veljovic 2017), the performance in this respect is currently not known. When the Mesinger and Veljovic (2017) solution is compared to Long's solution rather than with the still erroneous Klemp's improved solution, strong differences appear: the maximum of $u$ at the height $1.5 \mathrm{~km}$ has disappeared totally and a strong artificial maximum of $u$ at the surface appears. In the lee of the mountain and near the surface, the isolines of $u$ are strongly and artificially curved and the increase of $u$ in the lee of the mountain is absent. It is not known whether the practical consequences seen by Gallus (2000) are improved with the revised ETA model. For longer forecasts, Mesinger and Veljovic (2017) showed improvements of the revised ETA model.

Currently, new model designs involving new numerical schemes are mainly motivated by the requirements caused by new generations of massively parallel computers and requirements of computational efficiency, to a lesser degree by creating a better representation of lower boundary conditions. In one respect, cut-cells or comparable developments have an impact on model efficiency. With very high resolution, the numerical representations of mountains may become so steep that terrain-following models become unstable. This should not be expected with about $7 \mathrm{~km}$ resolution. Steppeler et al. (2006) showed cross-sections through the Alps with $7 \mathrm{~km}$ resolution where the mountain surface cut through more than one vertical layer in one cell. Also with the terrain-following control run, no stability problems due to mountain steepness were encountered at this resolution. For resolutions in the range of $1 \mathrm{~km}$, however, this may be a problem for stability. Zängl $(2002,2012)$ used model implementations involving horizontal extrapolations of the pressure field and could avoid instabilities due to mountain steepness in this way. This may be considered as a partial implementation of features of the cut-cell approximation, which helped to create an efficient treatment of steep mountains.

For a review of recent cut-cell developments see Steppeler and Klemp (2017). Currently realistic cut-cell models are implemented in low order. The scheme proposed by Steppeler et al. (2006) was implemented in a model of MM5 type. Such models request a strong numerical diffusion in realistic applications. The specific diffusion types used (hyper-diffusion Rayleigh damping, divergence damping) are described in Steppeler et al. (2003). The approximation order of such models is two for uncutcells, but can drop to first order for the cut-cells. It may even drop further to result in problems which can lead to noisy forecasts at the surface. The thin-wall approximation proposed by Steppeler et al. (2002) is able to avoid such problems, but must be applied to the non-advective terms only. The advection must be treated differently. The description of the thin-wall approximation was completed by Steppeler and Klemp (2017). Shaw and Weller (2016) discussed that noise was generated at the surface by cutcell discretization which was avoided by Steppeler and Klemp (2017). It remains to be said that currently no cutcell model in the framework of high-order finite difference method and local-Galerkin method exists. Existing realistic cut-cell models go back to old models of MM5 type.

For longer-range forecasts, it may be expected that the large-scale prediction could benefit from a better meso-scale forecast. The investigation of this effect is straightforward but would require considerable resources. A global version of LMZ would have to be created and a sufficiently large 
ensemble of forecasts should be evaluated. Additionally, some shortcomings of the LMZ would have to be removed. For example, the physical parameterization package would have to be modernized. The amount of research and programming capacity as well the required computation time would be considerable and is currently not available to the authors. Therefore, the proof of a positive impact of the cut-cells on 10-day forecasts cannot be carried out at this moment. In this paper, we use a simplified numerical experiment aimed to investigate just the sensitivity of a 10-day forecast to the cutcell discretization. Section 2 presents the model configuration and parameters employed in all test cases. Section 3 presents a series of results on 10-day forecasts on LMZ model and the standard Climate version of Lokal Modell (CLM, the national climate model of Germany from 2012). Finally, Sect. 4 concludes this study.

\section{Parameters and model configuration}

As the cut-cell model LMZ exists for limited area only, we investigate its impact on 10-day forecasts in the north Atlantic area using a limited large domain, shown in Fig. 1. A total of six cases for 10-day forecasts were done, using initial dates on 01 Jan (case A), 11 Jan (case B), 21 Jan (case C), 10 Feb (case D), 02 Mar (case E), 10 Mar (case F), 1989. The 10-day forecasts are hindcasts, as the lateral boundary values are observations, taken from data assimilation ERA 40 re-analysis of ECMWF. The ERA 40 re-analysis is also used for eyeball verification. While a more thorough investigation is desirable, the work presented is all which could be done within the means of the authors. This paper can only be a proof of concept and a first indication, as was pointed out before. In other respects, the model setup is described by Steppeler et al. (2003), where a graphic representation of the vertical resolution is also given. The horizontal resolution is about $7 \mathrm{~km}$. A rough estimate for the maximum steepness is 0.45 for LMZ and 0.25 for CLM. The different values are caused by the fact that CLM needs a filtered orography to obtain a reasonable performance (see Steppeler et al. 2006). A description of LMZ is provided by Steppeler et al. (2006), where the increase of meso-scale forecast quality is also described using a set of 50 forecasts. After publication, another set of 50 forecasts was carried out, confirming the result of the first 50 cases. For some features, such as position of precipitation, the increase in forecast quality was rather systematic.

A problem with cut-cells is the potential occurrence of very small elements, which can lead to smaller time-steps. There are a number of counter measures which were to different degrees used here. Small cells can be combined with larger ones. Vertical advection is treated implicitly and the thinwall approximation of Steppeler et al. (2002) is used, where the latter must not be used for advection, but rather for the fast waves (see details in Steppeler and Klemp 2017). These approximations were taken over for the results described here. In Steppeler et al. (2006), it was possible to use $90 \%$ of the operational time-step used in the old version of LM (Lokal Modell, it is now called COSMO-model, the Consortium for Small-Scale Modelling) with the terrain-following coordinate.

It would be natural to use LM with the terrain-following coordinate as control run. Experiments using this terrainfollowing version are called NOZ in this paper. The LMZ uses an old version of physics from 2006. Steppeler et al. (2006) used the exact control run and, therefore, showed the improvement using the same physics for LMZ and the terrain-following control run in the 24-h range. For the longer forecasts reported by Steppeler et al. (2013), these errors became so large that most of the comparisons were done using CLM with its improved physics for control. The control run NOZ with the same physics scheme as used by LMZ showed monthly precipitation rates above $1600 \mathrm{~mm}$ for desert areas. As the precipitation forecasts on large areas and in the subtropics and tropics were extremely bad with terrain-following coordinates, this old version of LM (now COSMO) could not be used as control. Instead a newer version of physics from 2012 was used as control. This was the
Fig. 1 The computational area for the forecasts. The orographic height (Unit: $\mathrm{m}$ ) is shown

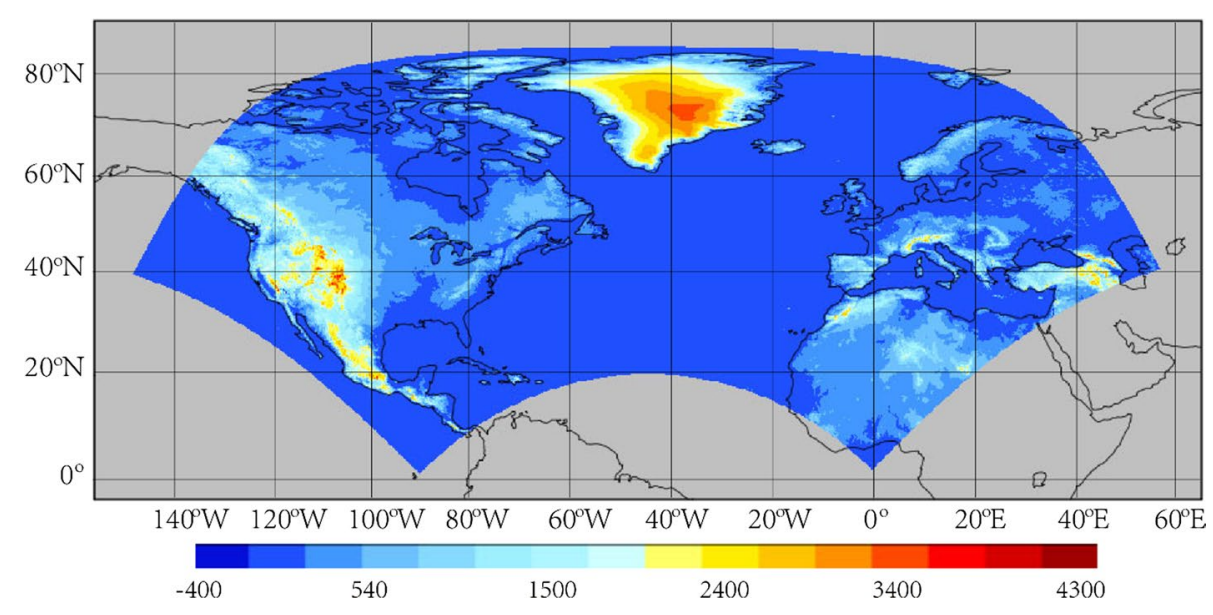


most up to date version of CLM in 2014, when the experiments were carried out. This puts the LMZ at a disadvantage. In spite of this, it will be shown that for the forecast investigated, LMZ has an advantage in the 10-day range in respect of the position of the Atlantic low. Other features, such as the Azorean high showed advantages for CLM. This is consistent with the result found in Steppeler et al. (2013) for time-averaged flows: the positions of meteorological features were systematically better forecasted with LMZ, while amplitudes could sometimes be better predicted with CLM. The LMZ has branched off the old version of LM (now COSMO) after one year of operational use of the latter. A major retuning of the physics scheme was necessary to get reasonable local forecasts. As it turned out, LMZ worked well with the old physics scheme and in this respect was never modernized. It may be expected that a retuning of physics for LMZ would also benefit LMZ.

The experimental setup can be summarized as follows: the vertical structure of LMZ and the control CLM is the same as described in Steppeler et al. (2003). The experimental setup in the comparison between LMZ and CLM is similar as used by Steppeler et al. (2006), referred to as ST06 and also Steppeler et al. (2013), referred to as ST13. In the present paper, exact control run NOZ is not used, but rather CLM, as was also the case in ST13. CLM uses a physics scheme tuned to give a reduced amount of rainfall and the NOZ results are very bad. Therefore, the comparison to CLM is more meaningful as that to NOZ. As described in ST13, CLM uses a filtered orography, which now is standard for models using terrain-following orography. There is no need to filter the orography with LMZ and the results obtained in the present paper are obtained without filtering. Following the experimental setup used in ST06 and ST13, each model is used with the orography giving best results for this particular model, which means filtered orography for CLM and unfiltered for LMZ. ST06 shows examples and cross-sections through the Alpine mountains to indicate what this means in practice, also in respect of weather representation. As it is known, that steeper orography can result in better forecasts, some of the improvements by LMZ may be due to a more realistic mountain representation. However, with terrain-following coordinates unfiltered orography leads to unacceptable results (Steppeler et al. 2003).

The impact of cut-cells on the small scales and 1-day predictions was rather systematically investigated by ST06. The meso-scale improvements of ST06 were significant, as a rather large sample of cases was used. Rather, the impact on larger scales and longer forecast times is currently investigated only by sensitivity studies. As discussed above, ensemble of cases available is not sufficient to claim that the forecasts are generally improved. It may be reasonably expected that a systematic improvement in the prediction of small scales can lead to improvements in the larger scales. So here and in ST13 we can only claim the cases investigated are improved, which means that we have a sensitivity study.

For new developments, such sensitivity studies, even for single cases, can provide useful encouragement for further development of new methods such was the case with Mesinger et al. (1988) and Steppeler et al. (2003). ST13 provided longer forecasts for the positions of average rainfall and for this question a rather coarse resolution was sufficient. In the present paper, we investigate a longer 10-day forecasts concerning the scale of the highs and lows. We may reasonably expect an improvement, as the better meso-scale representation of flows (ST06) should after some time result into improvements in the larger scale. For such forecasts, a rather high resolution of $7 \mathrm{~km}$ is needed, which makes the experiments expensive. As for the average rainfall investigated by ST13, the improvements concerned the position of features, which seem to be affected by the representation of mountains. Some technical shortcomings of our experimentation are regrettable, such as the use of an old physics scheme for LMZ. However, for the special cases investigated, we obtain improvements in comparison with CLM, one of the up to date models at the time of experimentation.

\section{Results}

To illustrate the performance of the control run NOZ using the same physics as LMZ, the 10-day forecast from 1 Jan 1989 of NOZ is shown in Fig. 2, together with observation and the forecasts of CLM and LMZ. The fields are shown for Level 30, the same level as shown for the other fields shown. This approximately corresponds to $1000 \mathrm{hPa}$ for sea points. For all models discussed here, there are 31 levels counted from top to bottom. As this is over a large ocean area, the 10-day forecasts of both LMZ and CLM show a reasonable representation of the vertical velocity field, which is rather well-positioned for the case of LMZ. The CLM forecast has a stronger error. The position of the system is too much south and some features have a too strong amplitude. The NOZ forecast, using the same physics as LMZ with terrain-following coordinates has a totally wrong vertical velocity system with a wrong amplitude. The areas of strong up and downwind are too large. This appears to be caused by an amplification of errors which was avoided by the retuning of physics with CLM. Note that LMZ shows realistic values of frontal vertical velocities of a few $\mathrm{cm} / \mathrm{s}$. Because of this result and those shown in Steppeler et al. (2006, 2013), the LMZ results are compared with those of CLM, rather than NOZ.

In the present paper, it will be shown that the fast-moving Atlantic low, heavily influenced by the high orography of Greenland, is better forecasted in position, while slow moving features, such as the Azores high, can be better predicted with CLM. The latter result is not too surprising, as the physics system of CLM was tuned to represent the amplitudes better, 


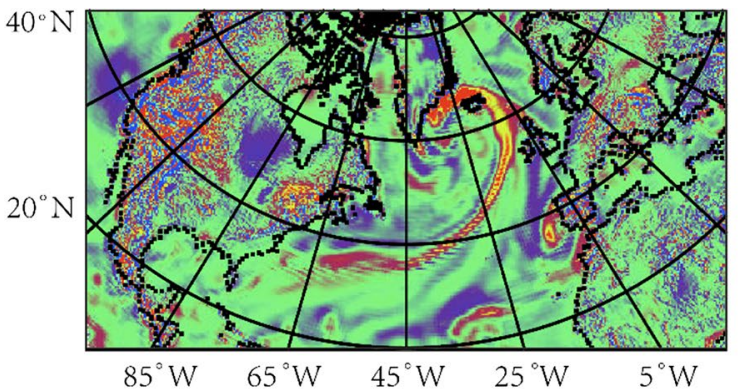

(a)

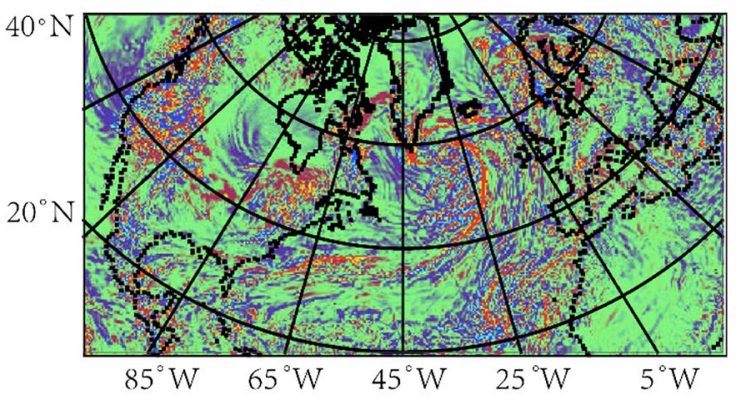

(c)

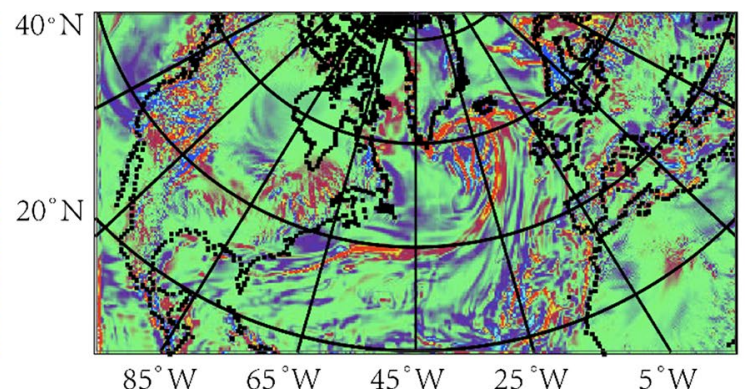

(b)

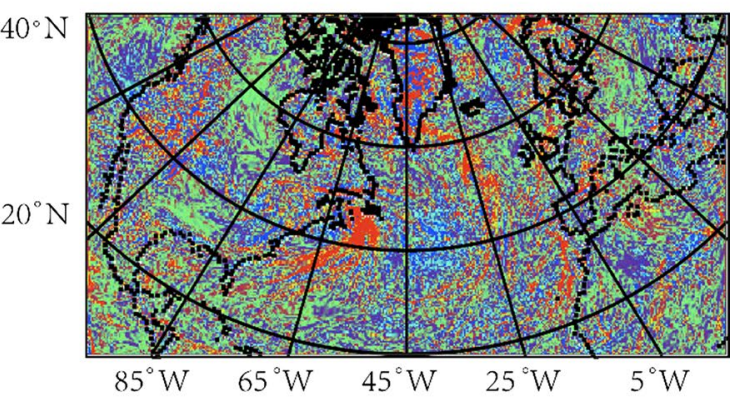

(d)
Fig. 2 10-day forecasts for the forecasted vertical velocity field $(\mathrm{m} / \mathrm{s})$. a Re-analysis, b LMZ, c CLM, $\mathbf{d}$ NOZ. NOZ is the exact control case for $\mathbf{b}$, using untuned physical parameterizations. The amplitudes for

while LMZ, using an old version of the physics system, does not enjoy the benefit of this development. Figure 3 shows the forecasted V-velocities for case A at day 6 . As illustrated in Fig. 1, a rotated latitude-longitude grid is used. The V-velocity is the poleward component of velocity in this rotated coordinate system. The position of the fronts belonging to a low south of Iceland can be seen. In this global plot of a highresolution forecast, they come out rather sharp. At this time the differences between the forecasts are not pronounced. In particular, the differences between LMZ forecast and control in the lee of Greenland cannot be interpreted to show a significant difference in phase. This finding is consistent with the fact that 6 days are within the useful forecast range of models of this type (Haiden et al. 2017). Any serious phase error at this forecast time would compromise this. The amplitudes of both forecasts are somewhat too high. Additionally, the velocities at the Spanish and African coasts are predicted too strong. For LMZ, this amplitude error is higher, consistent with findings of Steppeler et al. (2013).

Figure 4 shows the same result as Fig. 3 but for the 10-day forecast. Now the position of the Atlantic low and the frontal system are much better forecasted with LMZ. The frontal system of the Atlantic low is realistically predicted with LMZ to reach Iceland, while CLM did it much south. A second frontal system north of Iceland is formed with CLM, but not
$\mathrm{NOZ}$ are much too high and the vertical velocity structure belonging to the low can hardly be recognized

with LMZ. This second unrealistic frontal system is associated with an artificial low, which is formed with CLM, but not with LMZ.

Figure 5 shows the forecasted perturbation of pressure $p^{\prime}$ at Day 10. There are differences between the forecasts and we consider only the positions of lows and fronts, in particular in the Atlantic, where the orography had a considerable impact on the 10-day development, as Greenland is in the way of the principal trajectories of lows in particular. The positions of lows are better predicted with LMZ. For amplitudes there is no such systematic difference. CLM has formed an unrealistic second low north of Iceland, which realistically is missing with LMZ.

In this case the prediction of the position of the Atlantic low is better with LMZ after 10 days. We have a total of six cases confirming this finding. Amplitudes did not improve with LMZ and were sometimes better with CLM. This shows an interesting sensitivity of the 10-day forecast to the cutcell discretization. For a proof of forecast improvement, the ensemble is not large enough and the model setup is not sufficient. Figure 6 shows $p^{\prime}$ for all six cases A-F. There is a strong difference in the positions of the lows. The minima of the lows are marked and the difference between the position of a forecasted low to that of the observation is taken as the error and indicated in Table 1. For most cases, the LMZ 


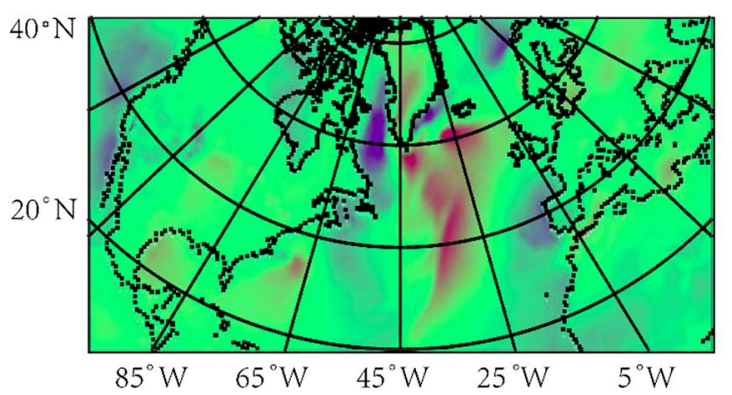

(a)

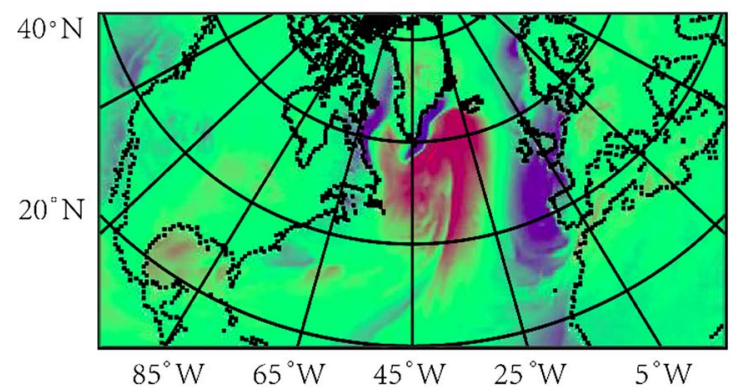

(b)

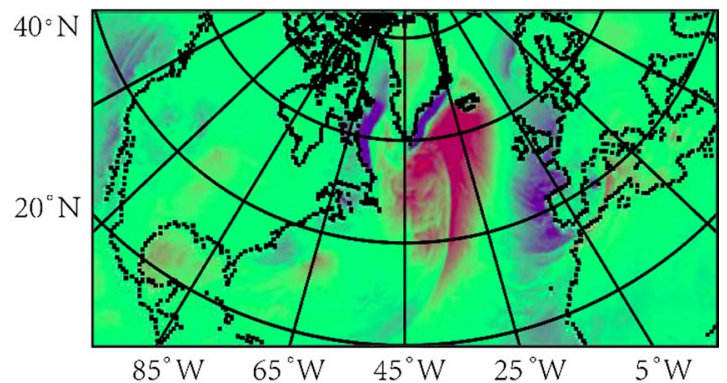

(c)

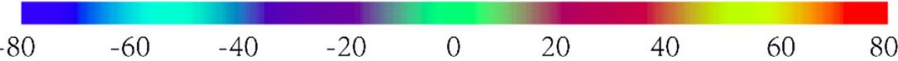

Fig. 3 Day 6 forcast of the V-velocity (m/s). a Re-analysis; b LMZ; c CLM

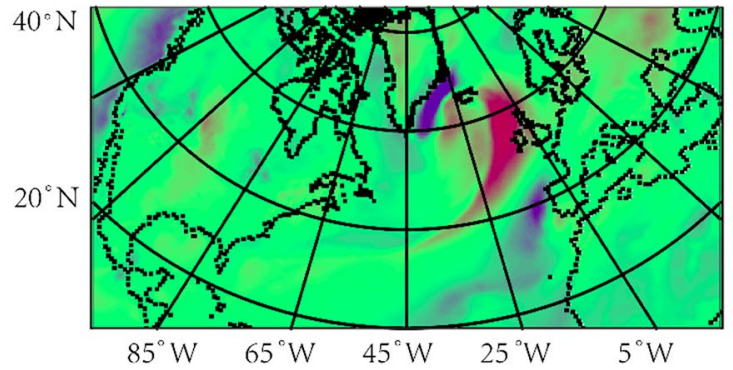

(a)

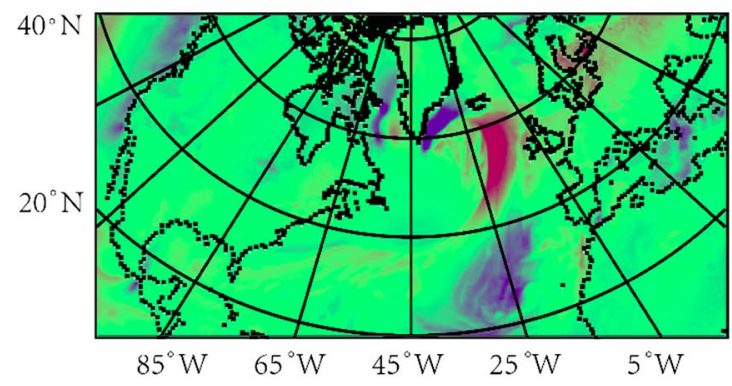

(b)

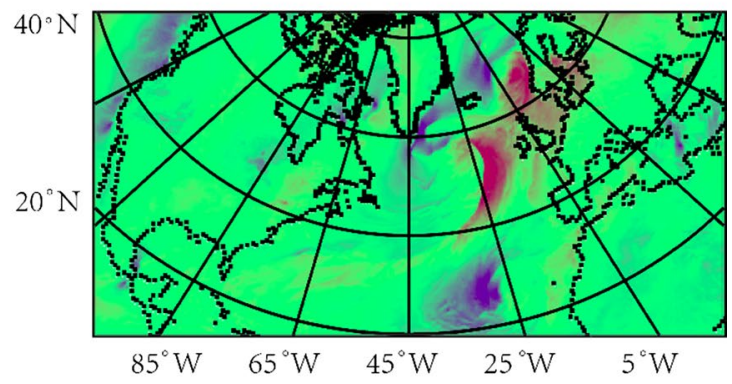

(c)

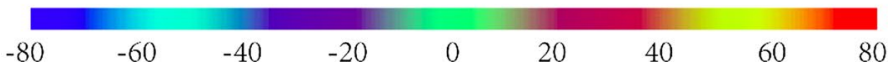

Fig. 4 As with Fig. 3 at day 10 forecasts 


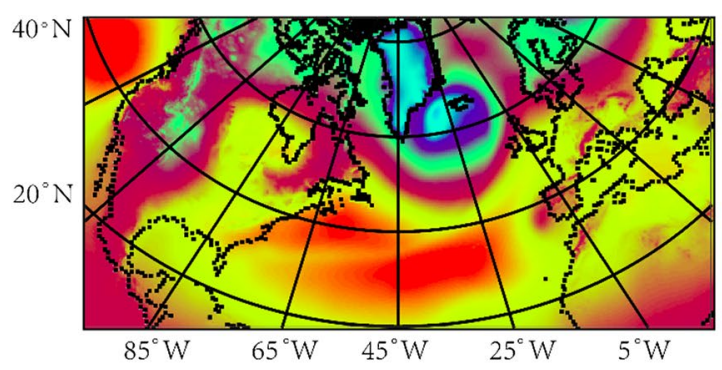

(a)

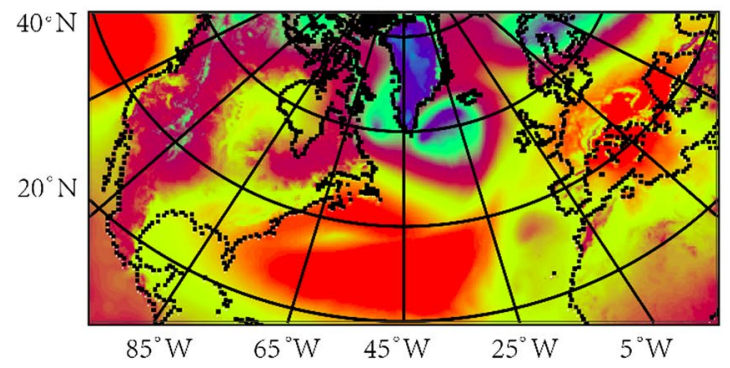

(b)

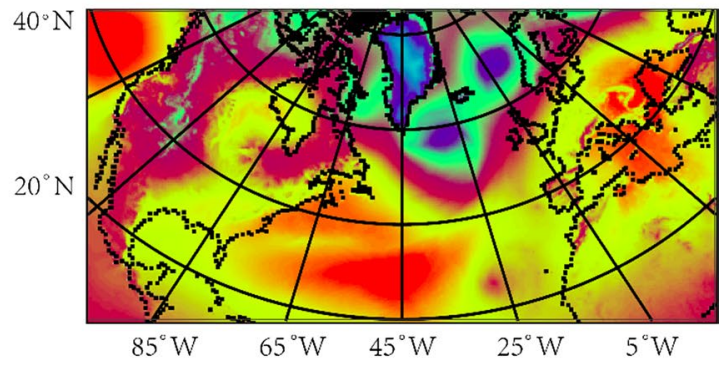

(c)

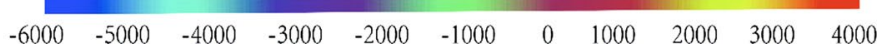

Fig. 5 The perturbation pressure $p^{\prime}$ (Unit: Pa) belonging to Fig. 3

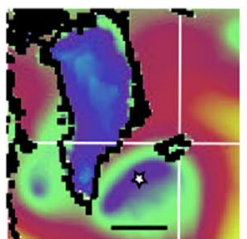

(a1)

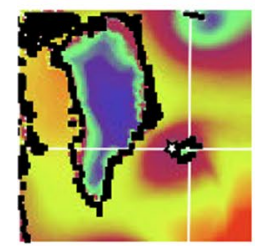

(b1)

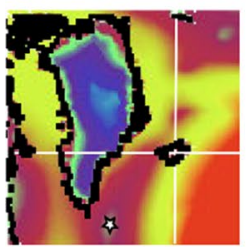

(c1)

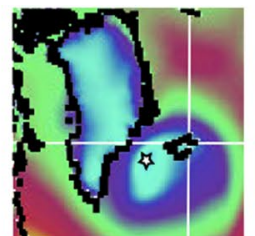

(a2)

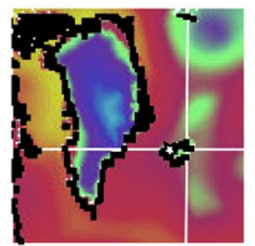

(b2)

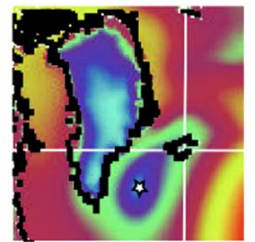

(c2)

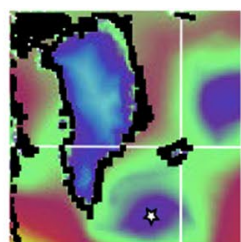

(a3)

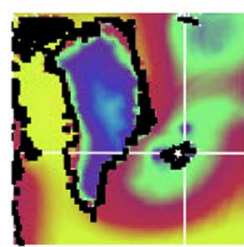

(b3)

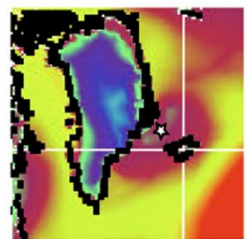

(c3)

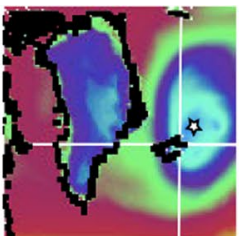

(d1)

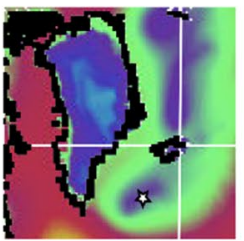

(e1)

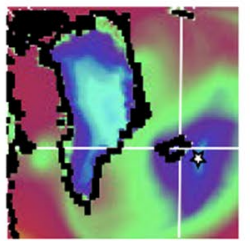

(f1)

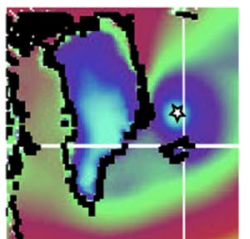

(d2)

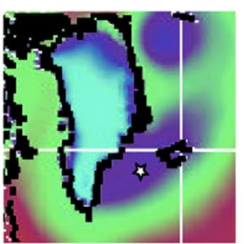

(e2)

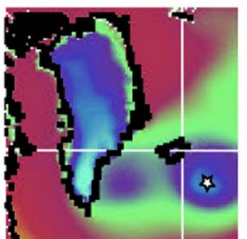

(f2)

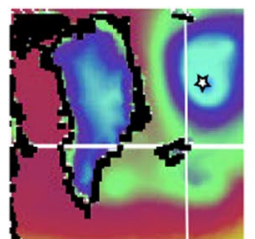

(d3)

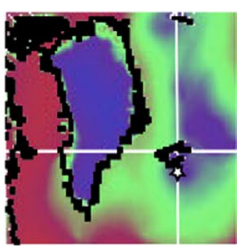

(e3)

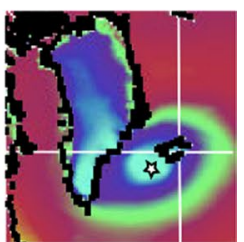

(f3)

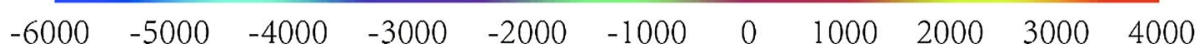

Fig. 6 The $p^{\prime}$ field (Unit: Pa) at day 10 forecasts for the cases A-F. The minima of lows are marked with stars for statistical verification. The first and fourth columns show LMZ forecasts, the second and fifth columns show observations and the third and sixth columns show CLM forecasts. The indicated co-ordinate lines cross at latitude $\left(65^{\circ} 27^{\prime} \mathrm{N}\right)$ and longitude $\left(16^{\circ} 36^{\prime} \mathrm{W}\right)$ in the unrotated lat-lon system. The arrow in (a1) indicates the length unit used. For the description of the verification procedure see Sect. 3 
Table 1 The position difference of forecast in CLM and LMZ to the observation

\begin{tabular}{lllllll}
\hline$E(k)$ & Case A & Case B & Case C & Case D & Case E & Case F \\
\hline LMZ & 0.34 & 0.02 & 0.7 & 0.48 & 0.5 & 0.4 \\
CLM & 1.1 & 0.24 & 1.2 & 0.6 & 0.7 & 1.06 \\
\hline
\end{tabular}

The length unit is shown in Fig. 6a1 with black color. It is related to the scale of the lows forecast is better positioned. In a number of cases, multiple lows occur. For case B, we have three lows for observation and CLM while only one for LMZ. For case D, the observation and CLM show two lows and LMZ only one. In case F, the observation has two lows and forecasts only one each. For evaluation, we use only one of these lows (marked with stars). In case B, the CLM forecast has the correct number of lows, but only one fits reasonably, which is the one included in Table 1. For case D, only one of the lows predicted with CLM fits reasonably which is the one chosen for Table 1. In case $\mathrm{F}$, the east low of the observation is chosen for Table 1 . Let $E(k)$ be the position difference of forecast $k$ ( $k$ represents the case) to the corresponding conservation. The errors $E(k)$ are tabulated in Table 1 . The average error $\bar{E}=\frac{1}{6} \sum_{k=1}^{6} E(k)$ is 0.82 for the CLM forecasts and 0.41 for LMZ. The variance $\sigma=\frac{1}{5} \sum_{k=1}^{6}[E(k)-\bar{E}]^{2}$ of the errors is also reduced. That is 0.136 for the CLM forecasts and 0.051 for LMZ.

\section{Conclusion}

In the 10-day forecast range, Atlantic lows are strongly influenced by the high orography of Greenland. The forecasted positions of Atlantic lows were sensitive to the numerical discretization. In this forecast range, the numerical discretization had a strong impact on the solution. For the six cases investigated, the position errors with the cut-cells were smaller or equal than those of the terrain-following model.

\footnotetext{
Acknowledgements No authors reported any potential conflicts of interest. Dr. Li acknowledges the support of China Postdoctoral Science Foundation (Grant no. 2016M601101) and National Key R \& D Program of China (Grant nos. 2016YFC1401705 and 2017YFC0209800). Dr. Fang acknowledges the support of the Innovation of the Chinese Academy of Sciences International Partnership Project (Grant no. Y56601M601) and the EPSRC grant: Managing Air for Green Inner Cities (MAGIC) (EP/N010221/1). Dr. Steppeler thanks NCAR for providing the computer time on the Yellowstone computer. The paper was made possible by a number of research visits of the first author at NCAR, financed by NCAR. The authors thank Dr. J. Klemp for his advice concerning Long's solution. Finally, the authors thank the editor as well as the anonymous reviewers for their valuable comments and suggestions.
}

Open Access This article is distributed under the terms of the Creative Commons Attribution 4.0 International License (http://creativeco mmons.org/licenses/by/4.0/), which permits unrestricted use, distribution, and reproduction in any medium, provided you give appropriate credit to the original author(s) and the source, provide a link to the Creative Commons license, and indicate if changes were made.

\section{References}

Adcroft A, Hill C, Marshall J (1997) Representation of topography by shaved cells in a height coordinate ocean model. Mon Weather Rev 125:2293-2315

Gallus WA (2000) The impact of step orography on flow in the ETA model: two contrasting examples. Weather Forecast 15(5):630-639

Gallus JR, Klemp JB (2000) Behavior of flow over step orography. Mon Weather Rev 128:1153-1164

Good B, Gadian A, Lock SJ, Ross A (2014) Performance of the cutcell method of representing orography in idealised simulations. Atmos Sci Lett 15:44-49

Haiden T, Janousek M, Bidlot J, Ferranti L, Prates F, Vitart F, Bauer P, Richardson DS (2017) Evaluation of ECMWF forecasts, including 2016-2017 upgrades. Internal report for ECMWF.

Klemp JB (2011) A terrain-following coordinate with smoothed coordinate surfaces. Mon Weather Rev 139:2163-2169

Leuenberger D, Koller M, Fuhrer O, Schär C (2010) A generalization of the SLEVE vertical coordinate. Mon Weather Rev 138:3683-3689

Li J, Zheng J, Zhu J, Fang F, Pain CC, Steppeler J, Navon IM, Xiao H (2018) Performance of Adaptive unstructured Mesh Modelling in idealized Advection cases over steep Terrains. Atmosphere 9:444

Li J, Steppeler J, Fang F, Pain CC, Zhu J, Peng X, Dong L, Li Y, Tao L, Leng W, Wang Y, Zheng J (2019) Meeting summary: potential numerical techniques and challenges for atmospheric modelling. B Am Meteorol Soc. https://doi.org/10.1175/BAMSD-19-0031.1 (Accepted)

Lock SJ, Bitzer H, Coals A, Gadian A, Mobbs S (2012) Demonstration of a cut cell representation of 3-d orography for studies of atmospheric flows over very steep hills. Mon Weather Rev 140:411-424

Mesinger F, Veljovic K (2017) Eta vs. sigma: review of past results, Gallus-Klemp test, and large-scale wind skill in ensemble experiments. Meteorol Atmos Phys 129:573-593

Mesinger F, Janjić ZI, Ničković S, Gavrilov D, Deaven DG (1988) The step-mountain coordinate: model description and performance for cases of Alpine lee cyclogenesis and for a case of an Appalachian redevelopment. Mon Weather Rev 116:1493-1518

Mesinger F, Chou SC, Gomes J, Jovic D, Bastos P, Bustamante JF, Lazic L, Lyra AA, Morelli S, Ristic I, Veljovic K (2012) An upgraded version of the Eta model. Meteorol Atmos Phys 116:63-79

Schär C, Leuenberger D, Fuhrer O, Lüthi D, Girard C (2002) A new terrain-following vertical coordinate for atmospheric prediction models. Mon Weather Rev 130:2459-2480

Shaw J, Weller H (2016) Comparison of terrain-following and cutcell grids using a nonhydrostatic model. Mon Weather Rev 144:2085-2099

Steppeler J, Klemp JB (2017) Advection on cut-cell grids for an idealized mountain of constant slope. Mon Weather Rev 145:1765-1777 
Steppeler J, Bitzer HW, Minotte M, Bonaventura L (2002) Nonhydrostatic atmospheric modelling using a z-coordinate representation. Mon Weather Rev 130:2143-2149

Steppeler J, Doms G, Schättler U, Bitzer HW, Gassmann A, Damrath U, Gregoric G (2003) Meso gamma scale forecasts by the nonhydrostatic models LM. Meteorol Atmos Phys 82:75-96

Steppeler J, Bitzer W, Janjic Z, Schättler U, Prohl P, Gjertsen U, Parfinievicz J, Damrath U (2006) Prediction of clouds and rain using a z coordinate non-hydrostatic model. Mon Weather Rev 134:3625-3643

Steppeler J, Ripodas P, Jonkheid B (2008) Third-order finite-difference schemes on icosahedral-type grids on the sphere. Mon Weather Rev 136:2683-2698

Steppeler J, Park SH, Dobler A (2013) Forecasts covering one month using a cut cell model. Geosci Model Dev 6:875-882

Steppeler J, Li J, Fang F, Zhu J, Ullrich PA (2019) o3o3: a variant of spectral elements with a regular collocation grid. Mon Weather Rev 147(6):2067-2082. https://doi.org/10.1175/ MWR-D-18-0288.1

Sundquist H (1967) On vertical interpolation and truncation in connection with the use of sigma system models. Atmosphere 14:37-52

Wallace JM, Tibaldi S, Simmons AJ (1983) Reduction of systematic errors on the ECMWF model through the introduction of an envelope orography. Q J R Meteorol Soc 109:683-717
Yamazaki H, Satomura T (2008) Vertically combined shaved cell model in a z-coordinate non-hydrostatic model. Atmos Sci Lett 9:171-175

Yamazaki H, Satomura T, Nikiforakis N (2016) Three-dimensional cut-cell modelling for high-resolution atmospheric simulations. Q J R Meteorol Soc 142:1335-1350

Zängl G (2002) An improved method for computing horizontal diffusion in a sigma-coordinate model and its application to simulations over mountainous topography. Mon Weather Rev 130:1423-1432

Zängl G (2012) Extending the numerical stability limit of terrain- following coordinate models over steep slopes. Mon Weather Rev 140:3722-3733

Publisher's Note Springer Nature remains neutral with regard to jurisdictional claims in published maps and institutional affiliations. 